\title{
Le développement de la robotique à l'école
}

\author{
Federica Minichiello
}

\section{OpenEdition}

Journals

Édition électronique

URL : https://journals.openedition.org/ries/5864

DOI : $10.4000 /$ ries.5864

ISSN : 2261-4265

\section{Éditeur}

France Education international

\section{Édition imprimée}

Date de publication : 1 septembre 2017

Pagination : 11-14

ISBN : 978-2-85420-615-9

ISSN : 1254-4590

\section{Référence électronique}

Federica Minichiello, «Le développement de la robotique à l'école », Revue internationale d'éducation de Sèvres [En ligne], 75 | septembre 2017, mis en ligne le 01 septembre 2017, consulté le 24 juin 2021. URL : http://journals.openedition.org/ries/5864 ; DOI : https://doi.org/10.4000/ries.5864

Ce document a été généré automatiquement le 24 juin 2021.

(c) Tous droits réservés 


\title{
Le développement de la robotique à l'école
}

\author{
Federica Minichiello
}

1 Le Parlement européen a voté, en février 2017, le texte « Règles de droit civil sur la robotique »: parmi les décisions figure la création d'une Agence européenne pour la robotique et l'intelligence artificielle, pour pouvoir « relever les défis ouverts par le développement de la robotique ». Les progrès en termes de robotisation appellent à de nombreux questionnements, qu'il s'agisse de risques d'automatisation des emplois ou d'ingérence dans d'autres aspects de la vie courante : les robots représenteraient, à eux seuls, plus de $50 \%$ du trafic sur Internet ${ }^{1}$. La Fédération internationale de robotique [https://ifr.org] a estimé le chiffre d'affaire de la robotique "de service " (non industrielle) à 35 milliards de dollars, avec la France en deuxième position, en nombre de sociétés spécialisées dans le secteur. En éducation, les scénarios d'application de la robotique intriguent, tantôt objets d'enseignement pour une initiation intuitive à la programmation informatique, tantôt solutions d'accès à distance ou d'assistance aux enseignants, etc.

2 Cet article présente une sélection non exhaustive d'acteurs, de colloques et d'initiatives pour montrer comment la robotique trouve progressivement une place au sein de l'école, à l'instar du programme Youth Robotics en Arménie [ http:// www.armrobotics.am/eng], qui vise la création, d'ici 2019, de groupes de robotique dans toutes les écoles du pays (pour les 12-18 ans).

3 Sitographie arrêtée le 15 juillet 2017.

\section{Acteurs et initiatives}

\section{« L'usage de la robotique à l'école »}

4 Cet article de l'Agence des usages (TICE) offre un regard introductif sur la robotique éducative, loin d'être "juste une technologie à maîtriser "; son utilisation soulève des questions, comme le choix en termes de discipline et de scénarisation pédagogique, le 
rôle des enseignants dans la mise en œuvre des projets, l'impact réel sur les apprentissages. Trois catégories d'usage sont présentées: l'apprentissage de la robotique, dans des domaines comme la mécanique, l'électronique et l'informatique. L'apprentissage avec la robotique, basée sur l'interaction entre apprenants, enseignants et robots, compagnons pour les uns, assistants pour les autres; l'apprentissage par la robotique (par exemple, la construction de kits robotiques) de compétences transversales comme le travail collaboratif, le raisonnement scientifique, la résolution de problèmes. Si plusieurs études semblent confirmer un impact positif en termes d'apprentissages, la littérature existante serait souvent basée sur des rapports d'enseignants, avec une nécessité de confirmation par des études à plus large échelle.

[https://www.reseau-canope.fr/agence-des-usages/]

\section{«Robot et Éducation, de quoi parlons-nous ? "}

Ce colloque, organisé en mai 2016 par l'Institut français de l'éducation (IFÉ-ENS) et l'Université Lyon 2, explore les possibilités d'usage de la robotique dans un établissement scolaire en complément de ce qui existe déjà (environnements numériques de travail, logiciels, etc.) ; «l'affordance socio-culturelle» va notamment étudier la modification des comportements en salle de classe, engendrée par l'introduction d'un nouvel objet technologique ${ }^{2}$. Parmi les axes de développement exposés, on peut évoquer la télé-présence, avec un robot "avatar » de l'élève et de l'enseignant absent, ou " passerelle » entre l'école et l'extérieur - un expert externe, des musées hors horaires d'ouverture, des lieux lointains -, ainsi que les robots comme compagnons d'interaction sociale, capables de s'adapter à leurs interlocuteurs et d'en accompagner, de façon personnalisée, les apprentissages.

[https://clarolineconnect.univ-lyon1.fr/]

\section{Institut national de recherche en informatique et en automatique (INRIA)}

6 L'équipe Flowers de l'INRIA, spécialisée dans la robotique développementale, étudie par la modélisation informatique l'intelligence des enfants et leur aptitude à l'exploration spontanée. Depuis 2015, des colloques annuels sont organisés sur la robotique éducative, pour présenter les possibilités ouvertes par ces technologies, tout en reliant l'engouement actuel à une dynamique de plus long terme et des initiatives pionnières, comme le langage de programmation Logo dans les années 1970-1980. Parmi les projets, on peut citer les modules IniRobot de découverte de la robotique pour les enfants de 6 à 11 ans, pensés notamment pour les activités sur le temps périscolaire ; Poppy Education [https://www.poppy-education.org], une plateforme de ressources destinées aux collégiens et lycéens pour utiliser différentes versions du robot open source POPPY; dans le cadre du Programme d'investissement d'avenir 2, le projet PERSEVERONS [http://pi.espe-aquitaine.fr/perseverons], porté par l'Université de Bordeaux, pour mesurer l'impact des activités pédagogiques robotiques en termes de motivation, persévérance, décrochage.

[https://flowers.inria.fr/]; [http://dm1r.fr/roboeduc17/] 


\section{École polytechnique fédérale de Lausanne (EPFL)}

7 L'EPFL accueille le siège du Pôle de recherche nationale (PRN) robotique, réunissant sur une période de 12 ans les principaux acteurs suisses de la recherche en robotique (2010-2022). Parmi les réalisations de l'école, on peut citer le robot Thymio 2 [https:// www.thymio.org] et son logiciel open source de programmation graphique, couplé à une programmation textuelle simplifiée, utilisé pour le module IniRobot de l'INRIA cité précédemment. Un pilote avec le robot Thymio a été proposé à des élèves de seize écoles à travers le monde, pour simuler une mission collaborative d'exploration spatiale sur Mars. Cette expérience préliminaire encourageante est désormais ouverte à toutes les écoles volontaires en Suisse.

[https://www.nccr-robotics.ch/fr/];

[http://www.robotsenclasse.ch/]

\section{RÉseau de personnes-ressources au développement des compétences par l'Intégration des technologies de l'information et de la communication (RÉCIT)}

8 Le RÉCIT est un réseau québécois spécialisé dans l'éducation préscolaire, qui souhaite promouvoir l'intégration pédagogique des TICE par les enseignants. Il met à disposition de nombreuses ressources sur la robotique en école maternelle, comme des activités préparatoires - déplacements sur un quadrillage, construction en briques Lego - un jeu «oui/non» pour identifier les robots dans l'environnement avoisinant, un tableau comparatif des robots les plus répandus avec des informations très pratiques, comme la facilité d'utilisation, la nuisance sonore, les prérequis en termes d'espace. Le site propose également un recensement d'articles récents comme, par exemple, une entrevue en $2017 \mathrm{du}$ chercheur T. Karsenti (CRIFPE) sur l'utilisation du robot Nao avec des enfants atteints de troubles du spectre de l'autisme.

[http://recitpresco.qc.ca/]

\section{Robo-Scuola}

9 Le projet italien Robo-Scuola est un exemple, parmi d'autres, de déploiement de la robotique à l'école : 3000 élèves (entre 6 et 19 ans) et environ 100 enseignants, dans la région du Piémont, explorent l'application de la robotique dans des disciplines comme les mathématiques et l'art. Ils étudient, par exemple, les inventions de Léonard Da Vinci en essayant de reproduire le fonctionnement de ces machines, imaginées au $\mathrm{XV}^{\mathrm{e}}$ siècle, avec les technologies robotiques actuelles. Le cas de la région du Piémont offre également un exemple de partenariat public-privé (avec une fondation d'entreprise), pour ouvrir des espaces dédiés à des ateliers de robotique pour les écoles et deux laboratoires pour les enseignants, pour un accompagnement pédagogique à la préparation de cours avec les robots, en collaboration avec l'Istituto italiano di tecnologia. [https://www.iit.it];

[http://www.istruzionepiemonte.it/] 


\section{Robot-enseignant et initiation à la robotique (Corée)}

10 La Corée du Sud est souvent présentée comme un pays précurseur en robotique éducative : il y a dix ans déjà, on y répertoriait des expériences d'utilisation de robotsenseignants, comme le modèle iRobi, conçu pour accueillir des enfants en école maternelle et primaire. Ce marché s'est dissous depuis, en raison d'une demande insuffisante et d'une barrière d'accès en termes de prix. Le pays vise actuellement l'initiation à la robotique dès le collège, avec une dynamique en plein essor : un millier d'acteurs privés présents sur le secteur, environ 17 modèles de robots homologués par le ministère de l'éducation et de nombreux événements, comme l'olympiade internationale des robots.

[https://www.iroc.org] ${ }^{3}$;

[http://english.keris.or.kr]

\section{Human-Robot Interaction}

11 Ce portail est destiné à la recherche dans l'interaction homme-robot et à l'organisation d'une conférence annuelle qui en est à sa douzième édition. Parmi les dernières contributions, on peut citer des robots proposant des temps de pause sur mesure aux apprenants, particulièrement utiles pour des systèmes de tutorat interactif de jeunes élèves, avec des portées d'attention assez réduites. On y retrouve aussi l'EPFL, avec le prototype Cellulo [http://chili.epfl.ch/cellulo], dont l'ambition est «de devenir le papier/stylo de demain", révolutionnant une offre parfois bridée par des problématiques de prix, des difficultés logistiques ou d'intégration à des curricula existants. Cellulo est un robot portatif polyvalent, capable d'interagir avec de grandes feuilles de papier qui, littéralement, «contiennent » l'activité pédagogique; il peut devenir, au besoin, un globe que l'on déplace sur un planétarium pour étudier la variation de l'orbite, une molécule sur laquelle on analyse les effets causés par des vibrations et ainsi de suite.

[http://humanrobotinteraction.org/]

\section{NOTES}

1. https://www.incapsula.com/blog/bot-traffic-report-2016.html (2017).

2. Voir l'intervention et les travaux de S. Simonian, Institut des sciences et pratiques d'éducation et de formation de l'Université Lyon $2:$ http://recherche.univ-lyon2.fr/ecp/

3. Lire également: J. Yoo. "Results and Outlooks of Robot Education in Republic of Korea », dans Procedia - Social and Behavioral Sciences/176 (2015) téléchargeable de http:// www.sciencedirect.com et "Comment faire entrer les robots à l'école» (2016) https:// www.lesechos.fr/. 
INDEX

Mots-clés : robotique, programmation, interaction homme-machine

Palabras claves : robótica, programación, interacción hombre-máquina

Keywords : robotics, programming, human machine interaction

\section{AUTEUR}

\section{FEDERICA MINICHIELLO}

Federica Minichiello est chargée de veille au centre de ressources et d'ingénierie documentaires du Centre international d'études pédagogiques. Courriel : minichiello@ciep.fr 